\title{
Staple line bleeding control with monopolar cautery in laparoscopic sleeve gastrectomy
}

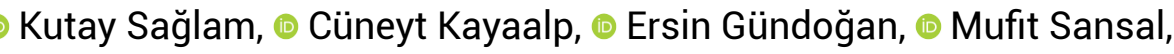 \\ (1) Aydın Aktaş, (1) Cihan Gökler, 아 Fatih Sümer \\ Department of Gastrointestinal Surgery, İnönü University Faculty of Medicine, Malatya, Turkey
}

\begin{abstract}
Introduction: During a sleeve gastrectomy, cauterization of the staple line for bleeding control is not recommended due to the worry of weakening the staple line. This study is an evaluation of sleeve gastrectomy outcomes in a cohort where all staple line bleeding control was achieved using monopolar cautery.

Materials and Methods: Between July 2014 and February 2019, monopolar cautery was used as the only method of staple line bleeding control following 187 sleeve gastrectomies. The rate of staple line leaks as well as intra-abdominal and intraluminal (gastrointestinal) bleeding was examined.

Results: A total of 144 women and 43 men were enrolled in this study (mean age: 34.1 years; mean body mass index: $43.6 \mathrm{~kg} / \mathrm{m}^{2}$ ). There were 5 leaks from the staple line (2.6\%) and 7 cases of gastrointestinal bleeding (3.7\%). There was no instance of mortality.

Conclusion: Staple line bleeding control with monopolar cautery during sleeve gastrectomy is a feasible, simple, inexpensive, and relatively safe method. Randomized trials are needed to compare monopolar cautery with other staple line bleeding control methods.

Keywords: Bariatric surgery; electrocautery; hemostasis; leakage; morbid obesity; stapled.
\end{abstract}

\section{Introduction}

Sleeve gastrectomy has become one of the most popular procedures in weight loss surgery. Technical simplicity, not requiring an anastomosis and absence of intestinal surgery are the main reasons for why surgeons and patients prefer sleeve gastrectomy. The most important intraabdominal complications of sleeve gastrectomy are staple line hemorrhage and leak, and surgeons are striving to minimize them using several techniques. Leak and bleeding rates following sleeve gastrectomy in the litera- ture ranges between $0-4.2 \%$ and $1.7-13.7 \%$, respectively. ${ }^{[1]}$ Using buttressing materials, fibrin glue, suturing, clipping and their combinations are the well-known methods for bleeding control and staple line reinforcement. In our practice, we do not prefer these techniques and instead only use monopolar cauterization for staple line bleeding control (SLBC) during both Roux-en-Y gastric bypass and sleeve gastrectomy. The aim of this study was to investigate (i) the efficiency of monopolar cauterization on SLBC and (ii) the rate of staple line leak following sleeve gastrectomy. 


\section{Materials and Methods}

Between July 2014 and February 2019, 187 patients underwent sleeve gastrectomy ( $n=167)$ or sleeve gastrectomy plus jejunoileal bypass $(n=20)$. A retrospective cohort study was designed and institutional ethical approval (2018/3-1) and international research registration (Research Registry 3630) were obtained. Informed consent was obtained from all individual participants included in the study. All patients had a body mass index (BMI) $>40 \mathrm{~kg} / \mathrm{m}^{2}$ or $>35-40 \mathrm{~kg} / \mathrm{m}^{2}$ with accompanying obesityrelated comorbidities. Patients were evaluated by a team consisting of a dietician, psychiatrist, surgeon, and an endocrinologist before surgery.

Patients were operated on in supine position and legs were in a closed position (feet together). Thromboembolism stockings were worn by all patients during the surgery. Pneumoperitoneum was created by a Veres needle from the upper left quadrant and $15 \mathrm{mmHg}$ was obtained. Three $12 \mathrm{~mm}$ and two $5 \mathrm{~mm}$ trocars, one for the Nathanson liver retractor, were placed. The omentum was divided from the greater curvature of the stomach by Ligasure (Covidien, Mansfield, USA). A 36F orogastric tube was placed throughout the lesser curvature of the stomach. Transection was started $6 \mathrm{~cm}$ proximal from the pylorus. The first of two staples were green loads $(4.1 \mathrm{~mm})$ and others were blue loads (3.5 mm) (Endogia, 60-mm, Covidien, Norwalk, CT, USA or 60-mm Echelon, Ethicon Endosurgery Cincinnati, OH). SLBC was provided by monopolar electrocautery (40 Watt), which was connected to the endoscopic scissors. Staple lines were inspected under an increased arterial blood pressure over $140 \mathrm{mmHg}$. Lastly, a methylene blue test was performed to test for leakage and the specimen was extracted through the trocar site.

Low molecular weight heparin was given in the early postoperative period and lasted for four weeks. An oral liquid diet was started on postoperative day one and patients were discharged on day three in the absence of complications. Age, gender, BMI, American Society of Anesthesiologists (ASA) score, comorbidities, additional surgical procedures, staple line leaks, intraabdominal and intraluminal (gastrointestinal) bleedings, and length of hospital stay were analyzed. For statistical analysis, descriptive methods were used.

\section{Results}

Of a total of 187 patients, 167 underwent sleeve gastrectomy and 20 underwent sleeve gastrectomy plus a jejunoileal bypass. 144 patients (77\%) were female, the mean patient age and BMI were 34.1+10.2 years and $43.6+4.8 \mathrm{~kg} / \mathrm{m}^{2}$, respectively. Twenty-four patients had hypertension and 31 had type 2 diabetes mellitus. Twenty-six patients had an ASA score of 3 and the others were considered a 2. Additional surgeries were required in five patients (three cholecystectomies, one adrenalectomy and one hiatal hernia repair). There was one postoperative intraabdominal bleeding $(0.5 \%)$ and gastrointestinal bleeding was diagnosed in six (3.2\%) patients by melena and there was a drop in the hemoglobin level. Patients with gastrointestinal bleeding were treated by medical methods. One patient was re-operated on postoperative day three for postoperative bleeding with staple line leak. There were five $(2.6 \%)$ staple line leaks both just below the esophagogastric junction. Four the leaks were managed by converting the sleeve gastrectomy to Roux-en-Y gastric bypass, ${ }^{[2]}$ and one of them treated with hyperalimentation and antibiotics methods. The mean length of hospital stay was $3.7+2.8$ (median=3, range: $3-25$ days) days. All SLBCs were achieved by monopolar cautery successfully and no additional intervention (suturing, glue application, clipping, etc.) were required.

\section{Discussion}

Numerous reinforcement methods have been described for SLBC during sleeve gastrectomy. Although many surgeons are unwilling to touch the stapler line with an electrocautery, we did not hesitate to use this technique as we have twenty years of experience using this method during open surgery without any adverse effects. We transferred this experience to our laparoscopic Rouxen-Y gastric bypass and sleeve gastrectomy techniques and here we analyzed our sleeve gastrectomy results with this technique. This study demonstrated that the intraabdominal bleeding and leakage rates $(0.5 \%$ and $2.6 \%$, respectively) were consistent with the sleeve gastrectomy literature.

Various methods (oversewing, fibrin sealant application, use of buttressing material) have been applied to the staple line in sleeve gastrectomies. All these techniques or materials aimed to prevent leakage and bleeding from the staple line, as well as known. Long staple line is responsible of the bleeding, intra luminal bleeding is uncommon and some series reported the reoperation rates because of intrabdominal bleeding as $1.4 \%$. ${ }^{[3,4]}$ The effective method aimed to prevent bleeding and degreased 
the leak rate. Bleeding control methods commonly aimed to control hemorrhage in the extra luminal origin staple line, clipping, fibrin sealant application or monopolar cautery like us. Mercier et al. ${ }^{[5]}$ compared effect of surgical glue with no staple line reinforcement method group and they find no differentiation on bleeding and leak rates. Coskun et al. ${ }^{[1]}$ reported that fibrin glue application on staple line, is an extraluminal bleeding control technique, zero leak and $0.3 \%$ bleeding rate. Musella et al. ${ }^{[6]}$ noticed in a randomized study that fibrin sealant significantly reduced the bleeding (14\% vs. $2 \%$ ) but did not have any statistically significant effect on the leak $(6 \%$ vs. 0). Albanopoulos et al. ${ }^{[7]}$ compared buttress material (Seamguard) and oversewing in a randomized control study and reported that oversewing prolonged the operation time and buttressing increased the cost. In that study, the staple line leak rate in the buttressing group was $4.2 \%$ and the bleeding rate was $2 \%$. Dapri et al. ${ }^{[8]}$ found that reinforcing the staple line with the buttress material reduced bleeding but had no significant effect on the leakage rate (5\%). Contrary, using a randomized study, Carandina et al. ${ }^{\left[{ }^{[]}\right.}$showed no significant differences in the postoperative bleeding and leakage rates between the reinforced and no-reinforced groups with overall $2.3 \%$ leak and $0.8 \%$ bleeding rates.In a systematic review, Gagner et al. ${ }^{[10]}$ compared different methods and found the leak rates for an absorbable membrane, oversewing and no-reinforcement groups were $1.1 \%$, $2.0 \%$, and $2.6 \%$, respectively. D'Ugo et al. ${ }^{[1]]}$ compared five different reinforcement methods with no reinforced patients in a multicenter study. They concluded that reinforcement was better than no-reinforcement with the outcomes of bleeding and leak rates as with and without reinforced groups were $2.5 \%$ vs $4.8 \%$ and $0.9 \%$ vs $13.7 \%$, respectively. In a recent study, comparation of SLR and non -SLR SG groups, showed that SLR did not affect the leak rates, degreased the bleeding rates but extended the operation time. ${ }^{[12]}$

It seems that the impact of the reinforcement to the staple line on the leak is still a controversial issue but its effect on the bleeding is mostly positive. Our leak and intraabdominal bleeding rates with monopolar cautery were $2.6 \%$ and $0.5 \%$, respectively. When we compared this method to other extraluminal bleeding control methods it was feasible. We did not think that the cauterization improved the leak rates because both the leak site and the leak rate are compatible with the literature data.

\section{Conclusion}

Monopolar cauterization is a feasible and cheap method for SLBC during sleeve gastrectomy. Randomized trials are necessary to compare its reliability and efficiency with other techniques.

\section{Disclosures}

Ethichs Committee Approval: The study was approved by the Local Ethics Committee.

Peer-review: Externally peer-reviewed.

Conflict of Interest: None declared.

\section{References}

1. Coskun H, Yardimci E. Effects and results of fibrin sealant use in 1000 laparoscopic sleeve gastrectomy cases. Surg Endosc 2017;31:2174-9. [CrossRef]

2. Saglam K, Aktas A, Gundogan E, Ertugrul I, Tardu A, Karagul $S$, et al. Management of acute sleeve gastrectomy leaks by conversion to Roux-en-Y gastric bypass: a small case series. Obes Surg 2017;27:3061-3. [CrossRef]

3. Silecchia G, lossa A. Complications of staple line and anastomoses following laparoscopic bariatric surgery. Ann Gastroenterol 2018;31:56-64. [CrossRef]

4. Gagner M, Deitel M, Erickson AL, Crosby RD. Survey on laparoscopic sleeve gastrectomy (LSG) at the Fourth International Consensus Summit on Sleeve Gastrectomy. Obes Surg 2013;23:2013-7. [CrossRef]

5. Mercier G, Loureiro M, Georgescu V, Skalli EM, Nedelcu M, Ramadan $\mathrm{M}$, et al. Surgical glue in laparoscopic sleeve gastrectomy: An initial experience and cost-effectiveness analysis. J Eval Clin Pract 2017;23:614-9. [CrossRef]

6. Musella M, Milone M, Maietta P, Bianco P, Pisapia A, Gaudioso D. Laparoscopic sleeve gastrectomy: efficacy of fibrin sealant in reducing postoperative bleeding. A randomized controlled trial. Updates Surg 2014;66:197-201. [CrossRef]

7. Albanopoulos K, Alevizos L, Flessas J, Menenakos E, Stamou $\mathrm{KM}$, Papailiou J, et al. Reinforcing the staple line during laparoscopic sleeve gastrectomy: prospective randomized clinical study comparing two different techniques. Preliminary results. Obes Surg 2012;22:42-6. [CrossRef]

8. Dapri G, Cadière GB, Himpens J. Reinforcing the staple line during laparoscopic sleeve gastrectomy: prospective randomized clinical study comparing three different techniques. Obes Surg 2010;20:462-7. [CrossRef]

9. Carandina S, Tabbara M, Bossi M, Valenti A, Polliand C Genser L, et al. Staple Line Reinforcement During Laparoscopic Sleeve Gastrectomy: Absorbable Monofilament, Barbed Suture, Fibrin Glue, or Nothing? Results of a Prospective Randomized Study. J Gastrointest Surg 2016;20:361-6.

10. Gagner M, Buchwald JN. Comparison of laparoscopic sleeve gastrectomy leak rates in four staple-line reinforcement op- 
tions: a systematic review. Surg Obes Relat Dis 2014;10:71323. [CrossRef]

11. D'Ugo S, Gentileschi P, Benavoli D, Cerci M, Gaspari A, Berta $\mathrm{RD}$, et al. Comparative use of different techniques for leak and bleeding prevention during laparoscopic sleeve gastrectomy: a multicenter study. Surg Obes Relat Dis 2014;10:450-4.

12. Demeusy A, Sill A, Averbach A. Current role of staple line reinforcement in 30-day outcomes of primary laparoscopic sleeve gastrectomy: an analysis of MBSAQIP data, 20152016 PUF. Surg Obes Relat Dis 2018;14:1454-61. [CrossRef] 